\title{
Axial Fan Performance under the Influence of a Uniform Ambient Flow Field
}

\author{
Till Heinemann $(\mathbb{D}$ and Stefan Becker \\ Institute of Process Machinery and Systems Engineering, University of Erlangen-Nuremberg, Cauerstr. 4, 91058 Erlangen, Germany \\ Correspondence should be addressed to Till Heinemann; tillheinemann@web.de
}

Received 6 July 2017; Accepted 18 January 2018; Published 1 March 2018

Academic Editor: Tareq S. Z. Salameh

Copyright (C) 2018 Till Heinemann and Stefan Becker. This is an open access article distributed under the Creative Commons Attribution License, which permits unrestricted use, distribution, and reproduction in any medium, provided the original work is properly cited.

\begin{abstract}
In their application to air-cooled condensers, axial fans are often subject to the detrimental influence of ambient flow fields at their inlet or outlet. While effects have been investigated mostly under perpendicular cross-flow conditions on fans operating as part of an array in their target design point, this study aims at examining the integral influence of uniform ambient flow fields on a single axial fan over a wide operating range. For this purpose, a wind tunnel fan test rig has been designed and assessed. Multiple angles between uniform ambient flow field and fan axis are examined in their integral influence on the characteristic curve of two distinct industrial axial fans with varying inlet modifications. Increasingly with the fan flow rate, perpendicular inlet cross-flow was found to always have a detrimental influence on fan performance. The straight bladed fan reacted less sensitively than the forward skewed fan, and the adverse cross-flow influence could be reduced with an inlet guard grille and with short conical shroud extensions. Cross-flow at the fan outlet showed potential static fan pressure increases at low flow rates.
\end{abstract}

\section{Introduction}

With rising global energy demands and a growing use of solar power around the globe, thermal power plants are increasingly built in arid locations. For a steam turbine cycle process, water scarcity often yields air-cooled condensers (ACC) to be more sensible economically and ecologically than standard water-cooled condensers [1,2]. Different ACC designs exist [3] and the most standard solution bases on an A-type frame of condenser tubes mounted above a horizontal platform of large diameter axial fans [4]. Arrays of dozens or hundreds of ACC modules are usual [5].

Adequate and reliable cooling of the condenser is essential to optimum plant performance, and so is fan energy consumption itself [6]. Owen and Kröger computed a reduction of the overall ACC cooling flow rate of over $15 \%$ at $8 \mathrm{~m} / \mathrm{s}$ wind velocities, which increased the steam turbine backpressure by over 20\% [7], which signifies a serious reduction of total plant power generation. It has been shown that flow detachment at the periphery of the ACC banks is caused by cross-flow under the platform, which is induced by the center fans' intake and natural ambient winds [8-16]. This reduces volumetric effectiveness of the cooling fans of down to $70 \%$ at the ACC perimeter $[6,15,17-22]$.

Most of the available experimental and numerical research on the phenomenon is carried out for the applied technical case of an A-frame ACC array [10-12, 14, 22-25]. Early research on a single wall mounted fan was carried out by Thiart and von Backström $[23,24]$ and on a single fan mounted with its inlet to a pipe section at different angles by Stinnes and von Backström [26].

Although most of the cited works investigate the influence of induced cross-draft on perimeter fans at different platform heights, a comparison can be made of their findings to the uniform ambient flow field of the present work. Based on the Salta model [8], Fourie et al. [22] suggest an empirical curve for the influence of ACC platform height and additional ambient cross-flow on the fan flow rate. The study shows substantial differences in cross-flow influence on fan performance due to fan design, and it points out the importance of fan position in the ACC array, that is, perimeter fan compared to center fans. A gradual decline of the system cooling flow rate was found, resulting in a decrease of around $20 \%$ at $9 \mathrm{~m} / \mathrm{s}$ ambient cross-flow velocity. This may serve as a reference 


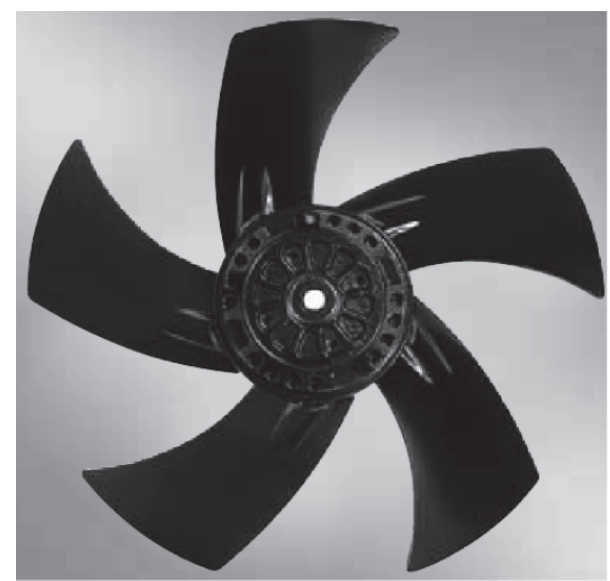

(a) Fan A

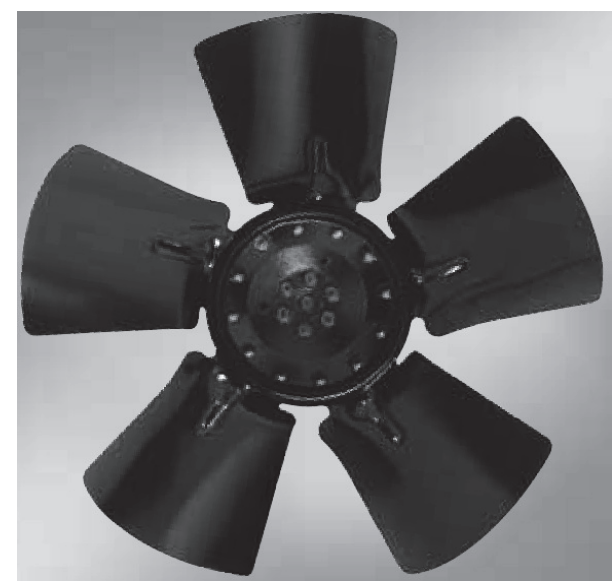

(b) Fan B

FIGURE 1: Test fans without shroud and guard grille.

figure to the results from the presented work's examination in perpendicular configuration. For individual fans at the ACC perimeter, decreases of $60 \%$ to $80 \%$ are reported in [22], but they are subject to strong additionally induced drafts from the center fans. Unfortunately, the results and empirical model by Fourie et al. do not translate well to the configuration of this work's approach, where no platform height exists.

The approach of the paper at hand extends the available information by examining single isolated axial fans in an ambient flow environment at various operating points. Thus, the influence of different angles $\gamma$ between fan axis and ambient flow field (velocity $U_{0}$ ) on fan pressure and efficiency curves is elaborated. The novelty of this approach lies in the experimental examination of cross-flow influence on an isolated single axial fan, while eliminating effects caused by neighboring fans and platform height. The presented work attempts to investigate integral fan behavior in a way more abstract from ACC application. It allows insight into fan performance under many different ambient flow field and installation conditions, over a wide range of flow rates (i.e., operating points).

This work aims at examining the influence of different ambient flow conditions at either fan inlet or fan outlet for a forward skewed industrial fan and a straight bladed fan. Installation conditions such as the presence of a concentric guard grille and the intake direction (motor side of the hub at inlet versus rotor side at inlet) are compared, and since shroud extensions have been reported to reduce negative cross-flow influence in ACC simulations [10, 12], the effect of different solid and porous cylindrical and conical inlet extensions is examined too.

\section{Experimental Setup}

2.1. Wind Tunnel Fan Test Rig. Inside a Göttinger type wind tunnel test section $\left(2.80 \mathrm{~m}\right.$ long, nozzle $\left.1.87 \times 1.40 \mathrm{~m}^{2}\right)$, the test fans shown in Figures 1 and 2, and described in Table 1, were mounted to a square duct section of $D_{\text {duct }} \times D_{\text {duct }}=300 \times$ $300 \mathrm{~mm}^{2}$ with either outlet in forced draft (FD) configuration
TABLE 1: Test fans manufacturer's specifications.

\begin{tabular}{lcc}
\hline & Fan A & Fan B \\
\hline Blade skew & Forward & None \\
$\xi_{\text {hub }}$ & $+20^{\circ}$ & $0^{\circ}$ \\
$\xi_{\text {tip }}$ & $+60^{\circ}$ & $0^{\circ}$ \\
Number of blades & 5 & 5 \\
$D_{\text {fan }}$ & $298 \mathrm{~mm}$ & $300 \mathrm{~mm}$ \\
Hub to tip ratio & 0.342 & 0.307 \\
$\dot{V}_{\max }$ & $0.947 \mathrm{~m}^{3} / \mathrm{s}$ & $0.767 \mathrm{~m}^{3} / \mathrm{s}$ \\
$f_{0}$ at $\dot{V}_{\max }$ & $45.0 / \mathrm{s}$ & $44.2 / \mathrm{s}$ \\
$P_{\sim}$ at $\dot{V}_{\max }$ & $230 \mathrm{~W}$ & $180 \mathrm{~W}$ \\
$\overline{\Delta p}_{\text {ts, } \max }$ & $200 \mathrm{~Pa}$ & $150 \mathrm{~Pa}$ \\
\hline
\end{tabular}

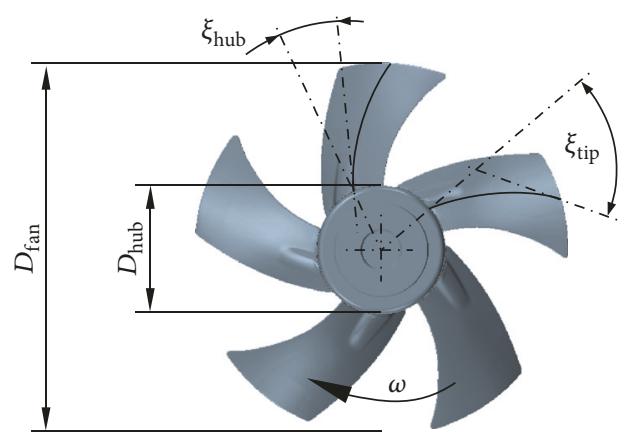

Figure 2: Test fan geometry (Fan A).

or inlet in induced draft (ID) configuration. The fan axis was located $1.5 D_{\text {duct }}$ above the test section ground. The experimental setup is shown in Figure 3, with the wind tunnel fan test rig (WFT) mounted to a rotatable platform to realize different angles $\gamma$ between fan and wind tunnel flow. Fan flow rate $\dot{V}$ was measured inside a settling chamber using multinozzles as described in ISO 5801 [27]. In Figure 3, the direction of $\dot{V}$ is indicated for FD operation, and it would be reversed for ID. 


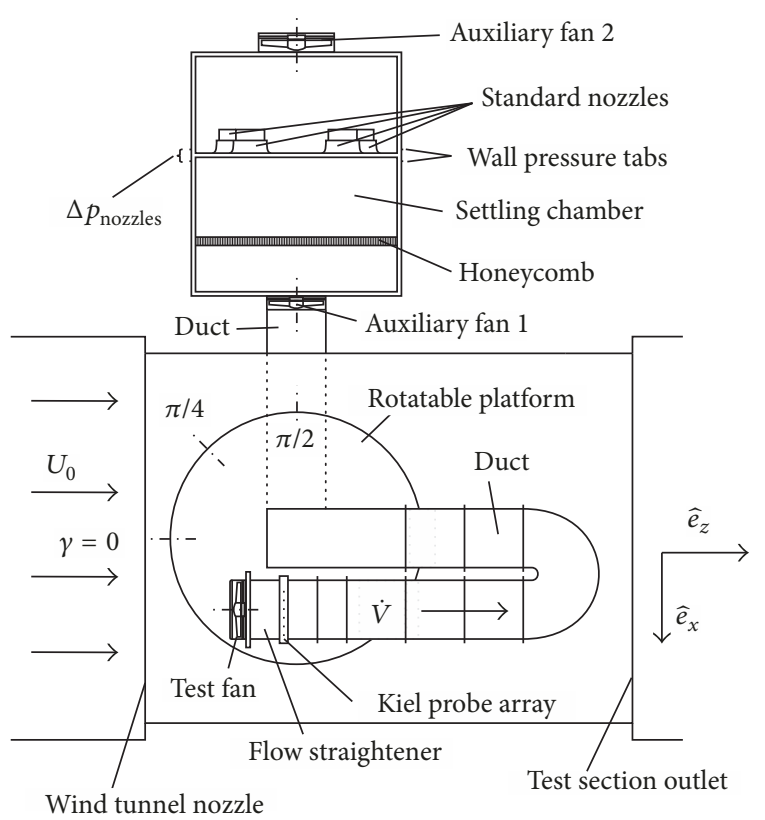

FIGURE 3: Wind tunnel fan test rig.

The test rig was designed to reduce wind tunnel blockage at different angles $\gamma$. To facilitate rapid total fan pressure measurements for a large number of operating points and configurations, a custom design array of $9 \times 9$ Kiel stagnation pressure probes as introduced in [28] was used inside the duct downstream of a $D_{\text {duct }} / 2$ long star shaped flow straightener (FS). This was necessary to reduce the swirl component at the fan outlet and realize flow directions below the critical onflow angles of the Kiel probes. The setup is shown in detail in Figure 4, where the manufacturer guard grille (GG) is in place at the fan inlet, fixing the hub's motor side to the shroud nozzle. This configuration is labeled FD-SI-GG, since the fan stator (motor side of the hub) is located at the inlet (SI), in forced draft operation (duct at fan outlet).

For the configuration without guard grille (noGG), Figure 4 also applies, with the notable difference that only four thin struts fix the fan motor to the shroud, instead of the sketched GG. A mirrored version of the fan with reverse operating direction design allows a rotor inlet (RI) configuration, where the rotating side of the hub is at the fan inlet.

In ID mode, the sketch in Figure 4 applies as well, with the following alternations: the fan flow direction $\dot{V}$ is reversed by $180^{\circ}$, and so is the orientation of the Kiel probe array. The absence of a considerable rotational velocity component in the duct, which means upstream of the fan inlet in ID also allows for the flow straightener to be omitted. With these three minor adaptations, Figure 4 illustrates the ID-SO-GG configuration, in which the free, motor side fan outlet is covered with a guard grille.

2.2. Assessment of Uncertainty and Comparability. Due to the spatial restrictions of the wind tunnel environment, the fan test rig described above was not in accordance with ISO 5801.

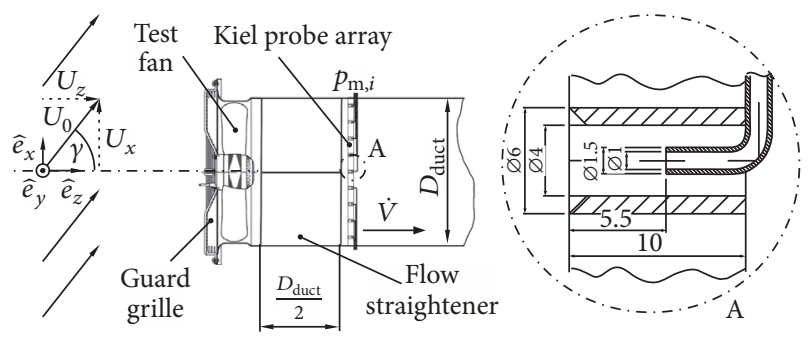

Figure 4: Measuring setup with Kiel probe array (FD-SI-GG).

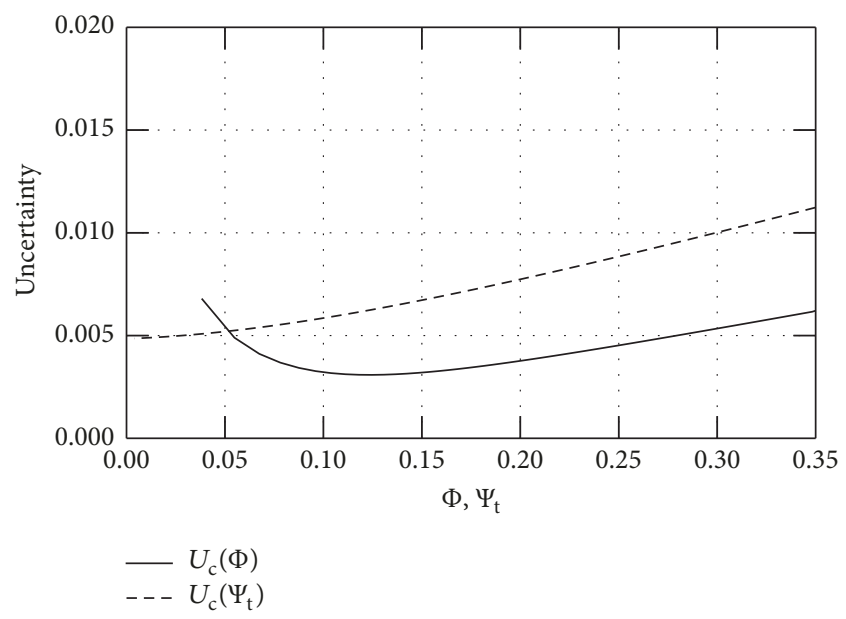

FIGURE 5: Expanded combined uncertainties of flow rate and total fan pressure coefficients (95\% level of confidence).

Figure 5 shows the extended combined uncertainties [29] of both flow rate coefficient

$$
\Phi=\frac{4 \dot{V}}{\pi D_{\text {fan }}^{2} \cdot\left(1-v_{\text {hub }}^{2}\right) \cdot u_{\text {tip }}}=\frac{\bar{c}_{z}}{u_{\text {tip }}}
$$

and total fan pressure coefficient (in FD)

$$
\begin{aligned}
\Psi_{\mathrm{t}} & =\frac{2}{\rho u_{\text {tip }}^{2}}\left[\left(\sum_{i=1}^{81} \frac{p_{\mathrm{m}, i}}{81}\right)-\frac{\rho}{2}\left(U_{0} \cdot \cos \gamma\right)^{2}\right] \\
& =\frac{2 \bar{p}_{\mathrm{m}}}{\left(\rho u_{\text {tip }}^{2}\right)}-\mu_{z}^{2},
\end{aligned}
$$

with blade tip velocity $u_{\text {tip }}=\pi f_{0} D_{\text {fan }}$ and the measured stagnation pressures over ambient pressure $p_{\mathrm{m}, i}$ at the $81 \mathrm{Kiel}$ probes (cf. Figure 4). The dynamic pressure caused by the ambient flow field is subtracted, introducing the ratio of wind tunnel velocity to blade tip velocity $\mu=U_{0} / u_{\text {tip }}$ and its axial projection $\mu_{z}=\mu \cdot \cos \gamma$.

As defined in [29], the expanded interval of uncertainty $U_{c}(\Phi)$ combines the individual measurement uncertainties of rotation rate $f_{0}$, density $\rho$, and static pressure difference over the multinozzles measured in accordance with ISO 5801, weighted by their linearized influence on $\Phi$. For the fan pressure coefficient, $U_{\mathrm{c}}\left(\Psi_{\mathrm{t}}\right)$ combines the uncertainties of $\rho, f_{0}, \gamma$, and $p_{\mathrm{m}, i}$ and the wind tunnel stagnation pressure 


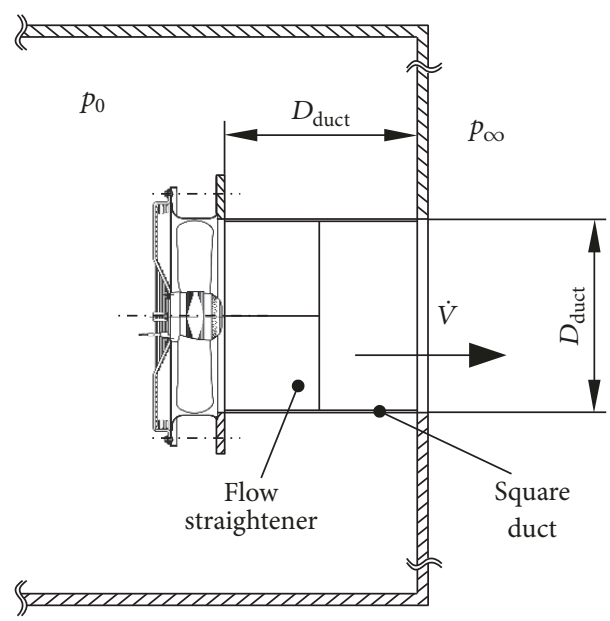

FIGURE 6: Standard fan test rig reference setup.

used to compute $U_{0}$. Uncertainties due to invariant fan geometries $\left(D_{\text {fan }}, \nu_{\text {hub }}\right)$ are not considered. The resulting values $U_{c}$ depend on the operating point and are shown in Figure 5 for a level of confidence of $95 \%$. It can be seen how the nonstandard capturing of fan pressure $\Psi_{\mathrm{t}}$ is less precise than $\Phi$, but the measuring setup and equipment are still considered sufficiently accurate for the performed parametric studies.

Besides the estimation of uncertainty, results measured in the WFT are compared to data gathered in a standard fan test rig (SFT) which meets the requirements of ISO 5801. Static fan pressure $\Psi_{s}$ from the free-inlet free-outlet SFT is compared to the static fan pressure estimate $\Psi_{s}^{*}$ gained by subtracting the area averaged fan flow rate $\dot{V}$ from measured stagnation pressure $\bar{p}_{\mathrm{m}}$ in WFT; that is,

$$
\begin{aligned}
\Psi_{\mathrm{s}} & =\frac{2\left(p_{0}-p_{\infty}\right)}{\rho u_{\text {tip }}^{2}}, \\
\Psi_{\mathrm{s}}^{*} & =\Psi_{\mathrm{t}}-\Phi^{2} .
\end{aligned}
$$

The static fan efficiency coefficients $\tilde{\eta}_{\mathrm{s}}$ and $\tilde{\eta}_{\mathrm{s}}^{*}$ are defined analogous to (3) with the product of fan pressure and flow rate scaled by measured electric power input $P_{\sim}$ :

$$
\widetilde{\eta}=\Phi \cdot \Psi \cdot \rho \pi D_{\text {fan }}^{2}\left(1-v_{\text {hub }}^{2}\right) \cdot \frac{u_{\text {tip }}^{3}}{\left(8 P_{\sim}\right)}
$$

with $\Psi=\Psi_{s}$ and $\Psi_{s}^{*}$, respectively. The SFT test setup is comprised of a $D_{\text {duct }}$ long duct section at the fan outlet, including the flow straightener, as shown in Figure 6. The systematic difference increases with remaining tangential and radial velocities $c_{\theta}$ and $c_{r}$ and with the axial velocity variance $\sigma_{c_{z}}^{2}$ over the duct cross section:

$$
\Psi_{\mathrm{s}}^{*}-\Psi_{\mathrm{s}}=\frac{\overline{c_{r}^{2}+c_{\theta}^{2}+c_{z}^{2}}-\bar{c}_{z}^{2}}{u_{\mathrm{tip}}^{2}}=\frac{\overline{c_{r}^{2}}+\overline{c_{\theta}^{2}}+\sigma_{c_{z}}^{2}}{u_{\mathrm{tip}}^{2}} \geq 0 .
$$

Figure 7 shows static fan curves from SFT and WFT for motor side inlet and guard grille configuration (SI-GG) and for free rotor side inlet configuration without a guard grille (RI-noGG). It was found that WFT and SFT results agree very well, yet WFT tends to underestimate the static fan pressure and efficiency curves slightly at flow rates $\Phi \geq 0.27$. Given the greater overestimation of $\Psi_{s}^{*}-\Psi_{s}$ in (5) at lower flow rates $\Phi$, it can be stated that lower values of fan pressure over flow rate in the order of $\Delta \Psi_{s} \leq 0.02$ were measured systematically in WFT compared to SFT. Deviations can be attributed to the differing operating and measuring setup. The developed WFT setup described above is deemed adequate for the quantitative estimation of the influence of the different parameters on fan performance curves under the influence of uniform ambient flow fields, as outlined in the Introduction.

\section{Integral Fan Performance}

With the WFT setup described and assessed above, the performance of the two axial test fans was examined in different configurations. The influence of ambient flow fields at the fan inlet (FD) with different angles $\gamma$ and ambient velocity ratios $\mu$ is described first, followed by the case of a flow field at the fan outlet (ID). Finally, different shroud extensions are assessed in their potential to reduce adverse flow field influence in FD on Fan A.

Tests were performed with constant wind tunnel velocities $U_{0}$, and the industrial fans' rotation rates $f_{0}$ showed moderate sensitivity to the operating point $\Phi$. The mean ratio of cross-flow to blade tip velocity $\bar{\mu}$ is the arithmetic mean over all operating points per data set.

3.1. Free Fan Inlet in an Ambient Flow Field. Because of its practical relevance, multiple combinations of direction $\gamma$, fan blade geometries (Fan A versus Fan B) and inlet configuration (RI-noGG versus SI-GG) were examined in FD configuration. The effect of $\bar{\mu}=0.25$ at different incidence angles $\gamma$ on the fan total pressure curves $\Psi_{t}(\Phi)$ is shown as measured in the WFT in Figures 8-10.

For the rotor inlet configuration of Fan A without guard grille shown in Figure 8, fan pressure was significantly affected by any direction $\gamma$ of the inlet ambient flow field. Even with the subtraction of stagnation pressure $\mu_{z}^{2}$, parallel onflow $\left(\gamma=0^{\circ}\right)$ caused an increase in fan pressure, and so did angles $\gamma \leq 56.3^{\circ}$. Further increment of $\gamma$ led to greater decrease of $\Psi_{\mathrm{t}}(\Phi)$ in an almost linear manner, in magnitudes of 0.04 to 0.08 . With the absolute reduction of total fan pressure being greater at high flow rates $\Phi$, the affected fan pressure curves are more inclined than the reference curve $(\mu=0)$. The respective change in efficiency $\widetilde{\eta}_{\mathrm{t}}$ is shown in Figure 11 . While also showing larger decreases at high flow rates at angles $\gamma \geq 56.3^{\circ}$, an increase in efficiency at acute angles $\gamma \leq 45^{\circ}$ was measured only at higher flow rates $\Phi>0.2$.

A comparison with results from the literature is difficult, since volumetric efficiency of the system is usually reported, which implies heat exchanger resistance characteristics. The drop of total efficiency $\tilde{\eta}_{\mathrm{t}}$ of about $43 \%$ at $\Phi=0.15, \gamma=90^{\circ}$, and $\mu=0.258$ from Figure 11 can best be roughly compared to the approx. $20 \%$ volumetric effectiveness decline found in [22] mentioned above, which was at $\mu \approx 0.27$ and a 


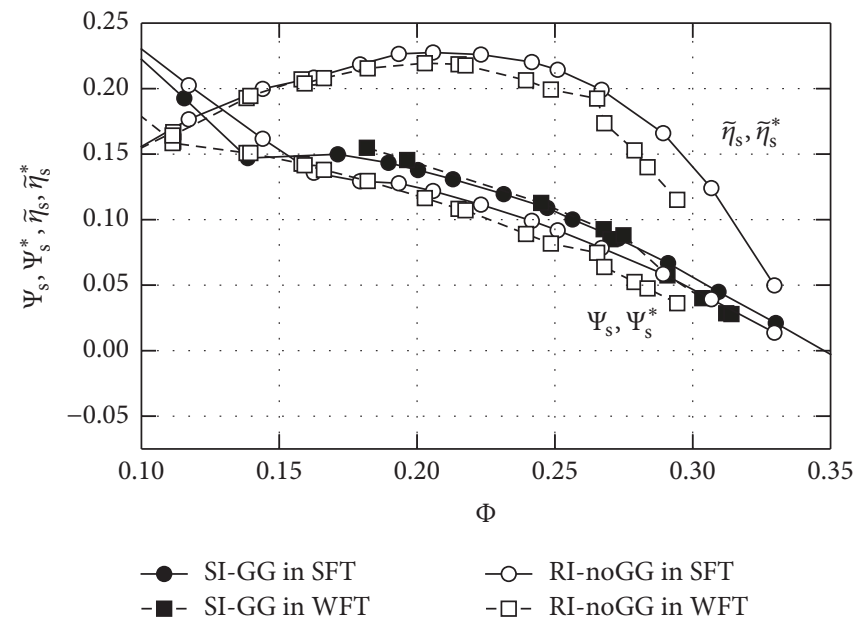

FIGURE 7: Comparison of static fan pressure and efficiency measured in standard fan test rig and wind tunnel fan test rig of Fan A in FD.

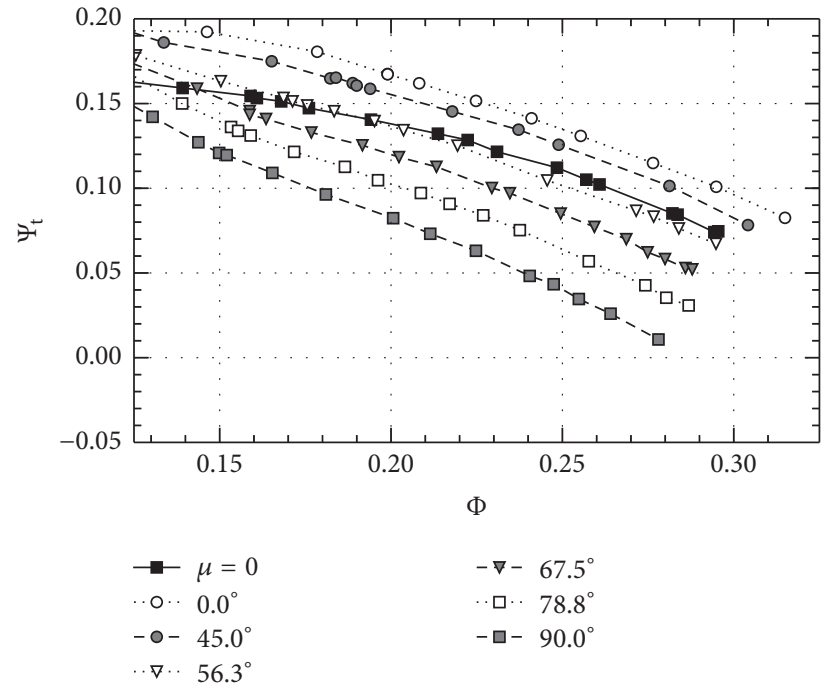

Figure 8: Fan A FD-RI-noGG total fan pressure at $\bar{\mu}=0.25$ and different $\gamma$.

lower operating point $\Phi \approx 0.1$. Even though comparable figures may be concluded for more similar $\Phi$, differences and variation in experimental and computational setup (fan design, ACC application and system characteristics, platform influence, operating point, etc.) of the works found in the literature still prevail. This interdicts any further quantitative comparison beyond the statement that, in general, results of a similar magnitude were found in this study.

In the configuration with the motor side of the hub and the guard grille at the fan inlet FD-SI-GG, the reference performance without ambient flow of Fan A was better than that in FD-RI-noGG. From Figure 9 it can be found that an inlet flow field with $\bar{\mu}=0.25$ showed much smaller influence on total fan pressure $\Psi_{\mathrm{t}}$ for any $\Phi$ and $\gamma$. Small gains in fan pressure were found for $\gamma \leq 45^{\circ}$ and $\Phi<0.25$. Greater angles $\gamma$ between flow and fan axis caused a reduction of $\Psi_{\mathrm{t}}(\Phi)$ in the range of 0.02 to 0.05 , also showing an increased inclination of

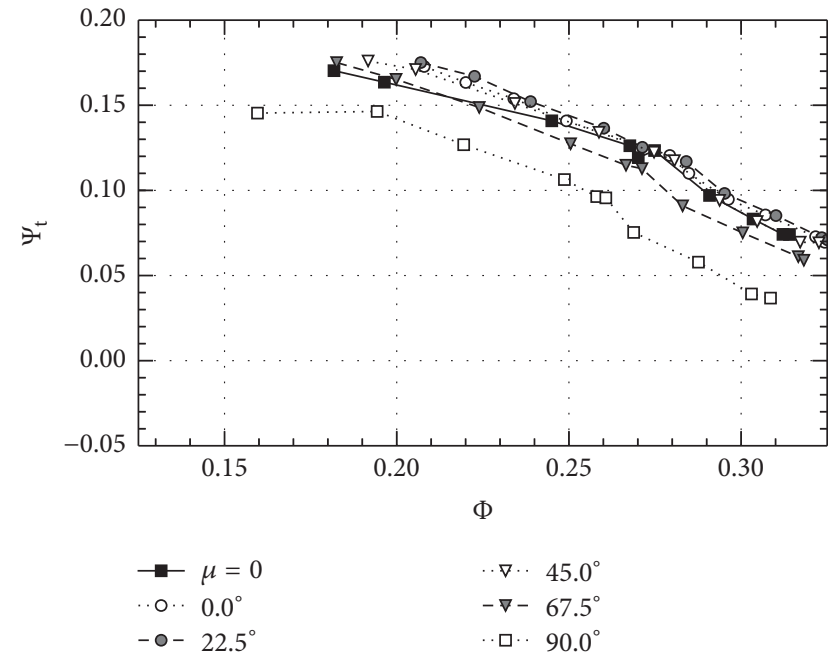

FIGURE 9: Fan A FD-SI-GG total fan pressure at $\bar{\mu}=0.25$ and different $\gamma$.

the fan curves, that is, a greater reduction of fan pressure at higher flow rates than at small ones.

The same favorable inlet configuration FD-SI-GG is examined for the straight bladed reference Fan B in Figure 11. All angles $\gamma \leq 45^{\circ}$ caused a considerable increase of the fan pressure at $\Phi<0.25$ and a moderate increase beyond that operating point. Only cross-flow of $\gamma=90^{\circ}$ was found to induce a substantial decline in fan performance. Again, all fan curves showed the characteristically more inclined shape compared to the reference curve $(\mu=0)$.

A decline in fan efficiency and pressure due to inlet cross-flow was to be expected, as summarized from results in the literature above. From there it is known that a flow detachment at the upwind edge of the fan shroud is common, which creates an asymmetric velocity distribution in longitudinal direction over the fan inlet. Even without this inlet vortex, the angle of attack at the fan blades varies laterally, with increases in relative velocities where fan blades 


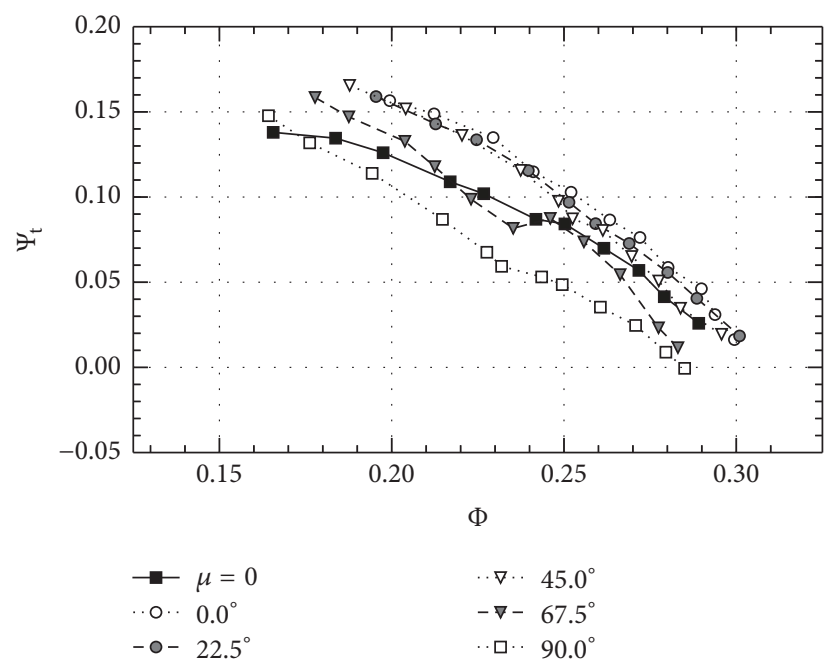

FIgURE 10: Fan B FD-SI-GG total fan pressure at $\bar{\mu}=0.25$ and different $\gamma$.

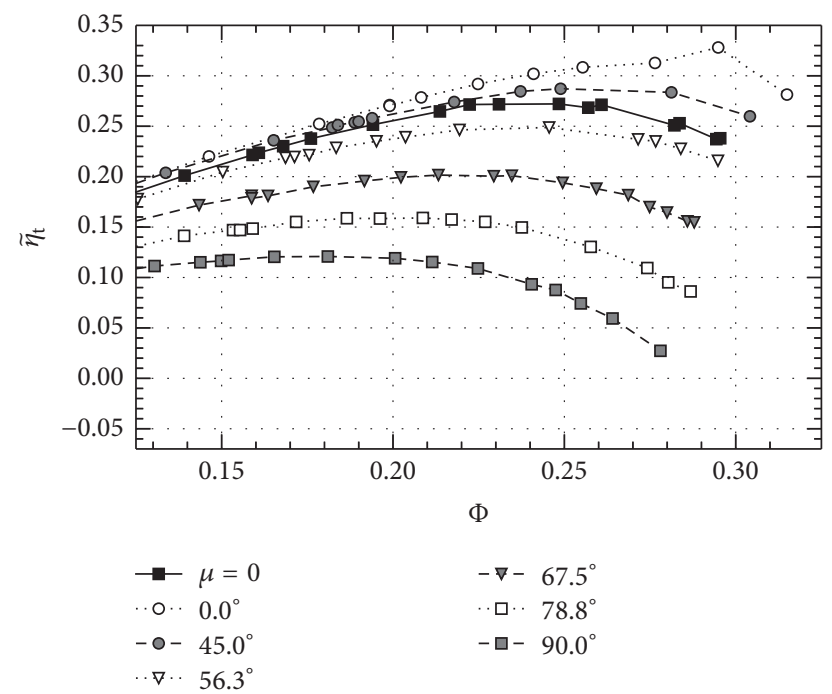

Figure 11: Fan A FD-RI-noGG total fan efficiency at $\bar{\mu}=0.25$ and different $\gamma$.

advance against $U_{0}$ and vice versa where they retreat. These two effects can be expected to result in a strong azimuthal variation of the radial circulation around the blades (see, e.g., [23]). This causes the flow to increasingly detach from the blades and losses to become greater. The radial flow component over the blades due to longitudinal inertia of the inlet flow field is believed to introduce further asymmetries and detachments, especially considering effects at hub and shroud.

Comparing FD-RI-noGG and FD-SI-GG results, the guard grille has been shown to reduce the influence of the inlet flow field, which can be attributed to its guiding and straightening effect on the inlet flow before it enters the rotor plane. The annular concentric guard grille is an obstacle especially to radial inlet velocity components.

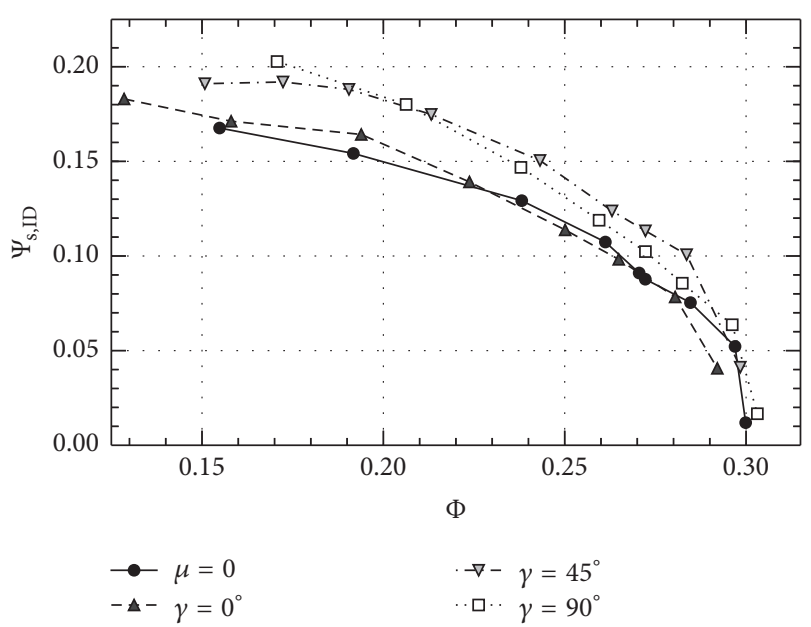

Figure 12: Fan A ID-SO-GG static fan pressure at $\bar{\mu}=0.25$ and different angles $\gamma$.

3.2. Free Fan Outlet in an Ambient Flow Field. Fan A was tested in ID configuration, that is, with its inlet mounted to the duct section and its outlet under the influence of an ambient flow field. As outlined above, the fan was fixed with a guard grille to the motor side of the hub at its outlet (SO$\mathrm{GG})$. Figure 12 shows the static fan pressure, computed as

$$
\Psi_{\mathrm{s}, \mathrm{ID}}=\mu_{z}^{2}-\frac{2 \bar{p}_{\mathrm{m}}}{\rho u_{\text {tip }}^{2}}=\left|\frac{2 \bar{p}_{\mathrm{m}}}{\rho u_{\mathrm{tip}}^{2}}-\mu_{z}^{2}\right| \text {, }
$$

with the measured averaged pressure difference $\bar{p}_{\mathrm{m}}<0$ between Kiel probes and static ambient pressure in the wind tunnel test section, assuming an outlet pressure increase by stagnation pressure $\mu_{z}^{2}$.

Parallel to the fan axis $\left(\gamma=0^{\circ}\right)$, the static fan pressure curve was hardly affected by the ambient flow directed against fan operating direction. Interestingly, a gain in static pressure was found for $\gamma=45^{\circ}$ and $90^{\circ}$ with decreasing flow rate $\Phi$. This may be caused by the effective (but not measured) lower pressure directly at the fan outlet caused by the detachment and deflection of the ambient flow around the fan outlet.

\subsection{Effect of Shroud Extensions on Adverse Inlet Cross-Flow} Influence. As shown in the literature above, inlet crossflow is a common effect in ACC fans with a detrimental influence on fan performance. The experimental findings presented in the previous sections confirm this effect in more detail for varying incidence angles $\gamma$, different fan design, and installation configurations. Based on positive influence shown by Duvenhage et al. [10] and Meyer [12], various inlet shroud extension designs were examined in their potential to reduce the negative cross-flow influence on the more stable FD-SI-GG configuration of Fan A, which was shown in Figure 9.

A total of seven different shroud extensions were manufactured from thin metal sheet material. The basic cylindrical and conical shapes are defined in Figures 13(b) and 13(c), differing in length to fan diameter ratio $\zeta$. A short solid 


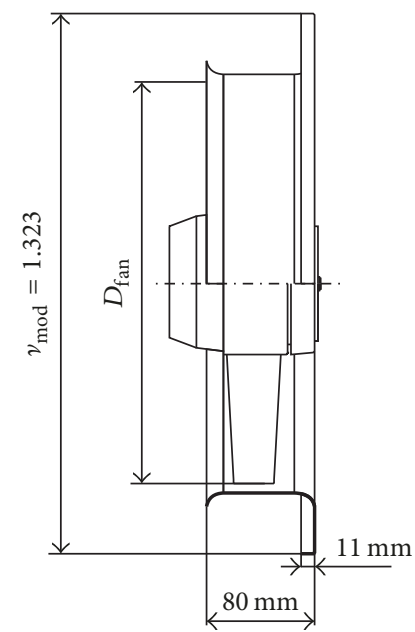

(a) REF

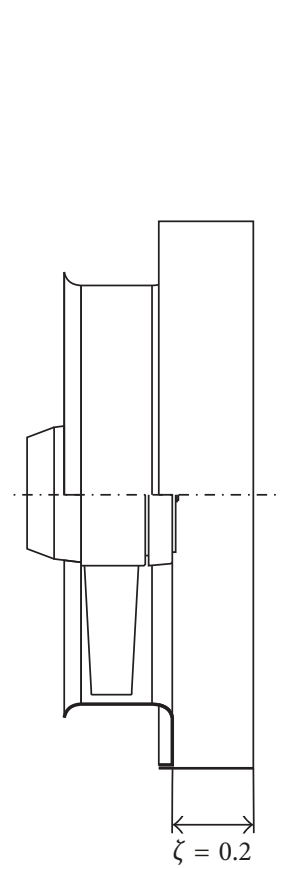

(b) CYL

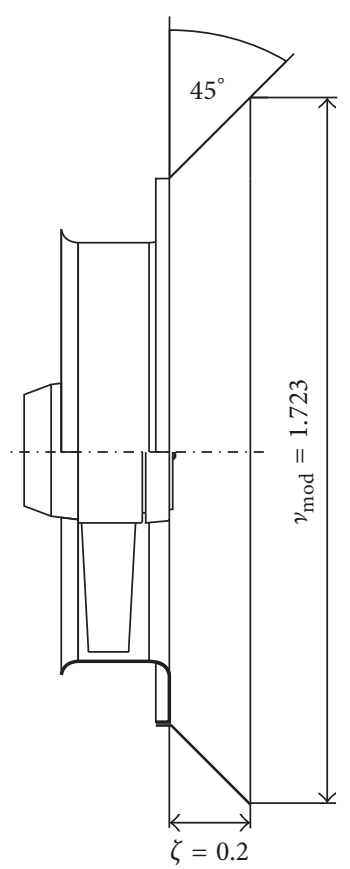

(c) $\mathrm{CON}$

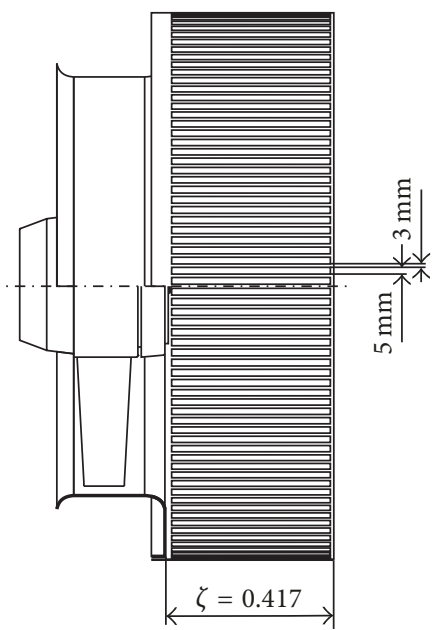

(d) POR-SL

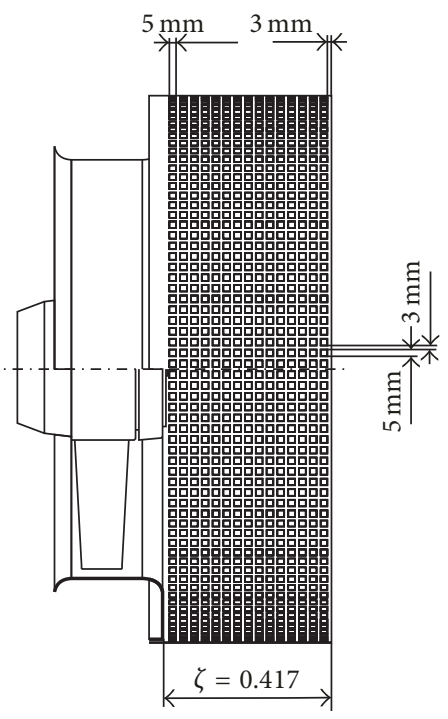

(e) POR-SQ

FIGURE 13: Basic geometries of inlet shroud extensions.

cylindrical inlet extension CYL-S and a longer one (CYL-M) were tested, with $\zeta=0.2$ and 0.417 .

Two cylindrical porous sheet designs with $\zeta=0.417$ were examined, denoted as POR-SL for a slot design shown in Figure 13(d), and POR-SQ for a square hole design illustrated in Figure 13(e). The respective solidity ratios of the sheets were 0.375 and 0.609 .

Three different solid conical inlet extensions were tested with $\zeta=0.2,0.417$, and 0.733 . The uniform aperture of $90^{\circ}$ resulted in different outlet diameter ratios $\nu_{\bmod }$ for the short, medium, and long conical devices CON-S, CON-M, and CON-L, listed in Table 2.
Reference results without ambient flow field $(\mu=0)$ are shown in Figure 14 for the cylindrical shroud extension and in Figure 15 for the conical ones. While all fan curves lie in proximity to the reference fan curve, some configurations already induced a detectable negative influence on $\Psi_{t}(\Phi>$ 0.26). In particular CYL-M showed detrimental reference performance.

Under the influence of cross-flow $\left(\gamma=90^{\circ}\right)$, any cylindrical device did clearly not improve fan performance, as it becomes obvious from Figure 16. Neither of the tested configurations CYL-S, CYL-M, POR-SL, and POR-SQ can be recommended as a good approach to reducing the adverse 


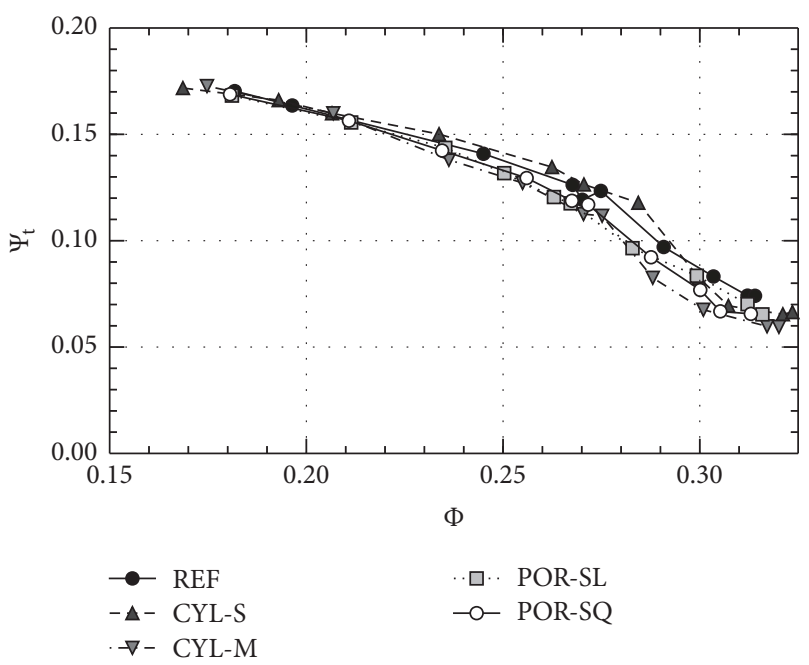

FIGURE 14: Influence of cylindrical shroud extensions $(\mu=0)$.

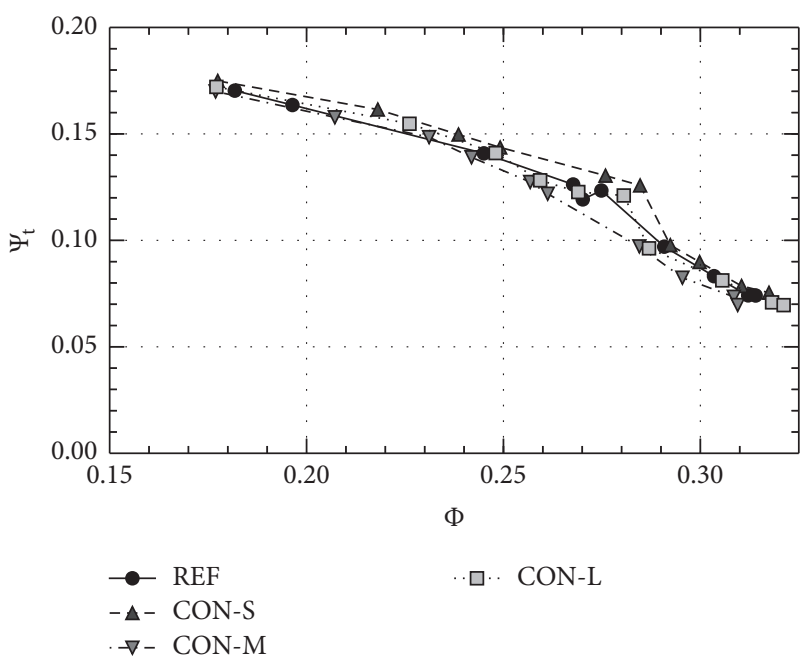

FIGURE 15: Influence of conical shroud extensions $(\mu=0)$.

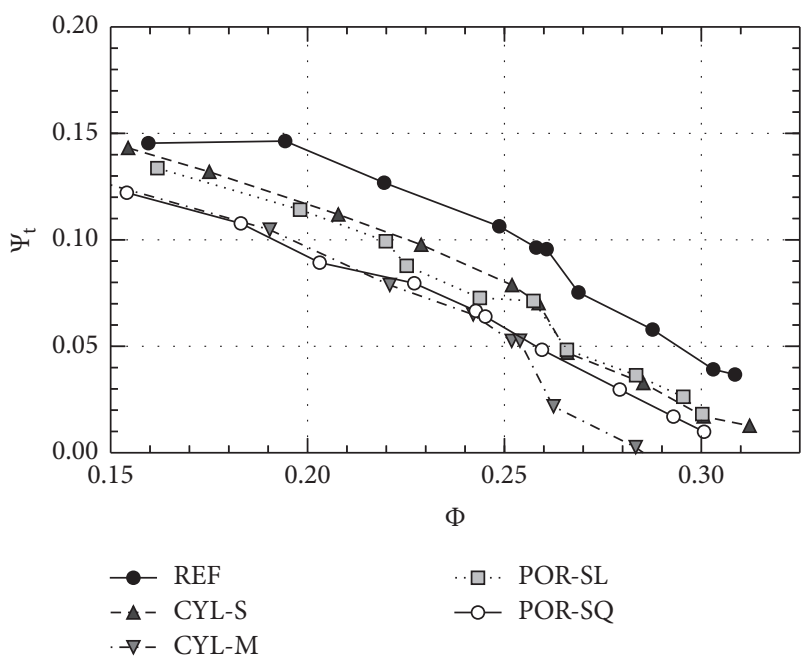

FIGURE 16: Influence of different cylindrical shroud extensions under $\bar{\mu}=0.245$ and $\gamma=90^{\circ}$.
TABLE 2: Geometric parameters of inlet shroud extensions.

\begin{tabular}{lccc}
\hline Name & Solidity & $\zeta$ & $\nu_{\text {mod }}$ \\
\hline REF & - & 0.0 & - \\
CYL-S & 1.0 & 0.200 & 1.323 \\
CYL-M & 1.0 & 0.417 & 1.323 \\
POR-SL & 0.375 & 0.417 & 1.323 \\
POR-SQ & 0.609 & 0.417 & 1.323 \\
CON-S & 1.0 & 0.200 & 1.723 \\
CON-M & 1.0 & 0.417 & 2.157 \\
CON-L & 1.0 & 0.733 & 2.790 \\
\hline
\end{tabular}

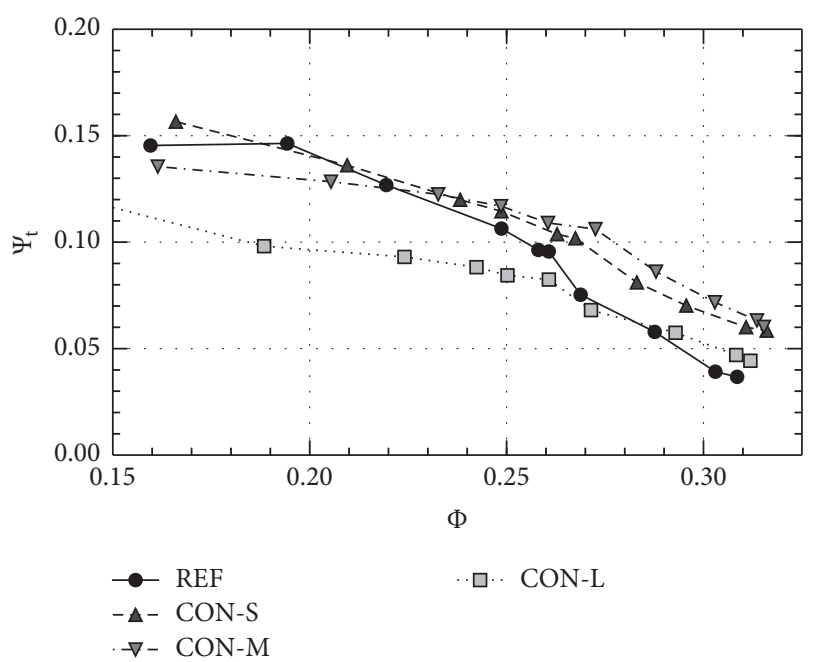

FIGURE 17: Influence of different conical shroud extensions under $\bar{\mu}=0.245$ and $\gamma=90^{\circ}$.

influence of inlet cross-flow compared to the given reference configuration.

Conical devices on the other hand did show a potentially beneficial influence on the fan performance under crossflow influence, as shown in Figure 17. At very high flow rates $\Phi>0.28$, all three extension sizes CON-S, CON$\mathrm{M}$, and CON-L increased total fan pressure, but below that the long conical device was not effective. Both the short and medium conical shroud extensions yielded higher fan pressures than the reference curve at $\Phi>0.22$, with CON$S$ performing better at lower flow rates and $\mathrm{CON}-\mathrm{M}$ at higher flow rates. Generally, a flattening of the total fan pressure curve $\Psi_{t}(\Phi)$ was achieved by the implementation of conical shroud extensions. It is believed that the flow detachment at the upwind edge of the shroud, which was documented, for example, in [12-16] simulations of inlet cross-flow, has less of a blocking effect at the fan inlet, because the vortex is more distant from the fan inlet axially and longitudinally (i.e., in wind direction). A more uniform flow field at the rotor inlet is the expected result of this, with consequently better fan performance. 


\section{Conclusions}

The influence of different uniform ambient flow fields at the inlet or outlet of industrial axial fans on their integral performance curves was investigated in a nonstandard fan test rig inside a wind tunnel test section. The fan test rig and total fan pressure measurements using an array of Kiel probes were assessed and in good comparison to results from a standard fan test rig.

In the practically most relevant case of a flow field at the fan inlet (FD), ambient flow fields caused an inclination of the fan curves at any angle relative to the fan axis. In a rotor inlet configuration without guard grille, great sensitivity to ambient flow was observed, with potential fan pressure gains at small angles between flow and fan axis but substantial reductions towards greater angles up to cross-flow. With the motor side of the hub and the guard grille at the inlet, fan curves varied much less due to uniform inlet flow fields. Compared to the forward skewed blade test fan, the straight bladed fan showed to behave much less sensitively to ambient flow, with relevantly lowered total fan pressure measured only in perpendicular cross-flow configuration.

A uniform ambient flow field at the fan outlet (ID) parallel to the fan axis hardly affected static fan pressure. Off-axis configurations up to cross-flow were found to even increase static fan pressure, especially at lower flow rates.

Possible performance gains with inlet shroud extensions were investigated in an inlet cross-flow configuration. Solid and porous sheet cylindrical devices showed detrimental influence, while short solid conical shroud extensions improved fan performance compared to the reference configuration, especially at high flow rates.

\section{Discussion and Outlook}

The presented test setup made a large quantity of parameter permutations possible, such that multiple aspects of ambient flow influence on fan performance could be investigated. The examination of cross wind influence could be extended from single operating point research to wide fan operating ranges. This resulted in the presented effect of increasingly adverse affection of total fan pressure with the fan flow rate due to cross-flow. But the rapid measurement procedure with the Kiel probe array has the disadvantage that the flow straightener's effect is always included in the measured characteristic curves, which is presumably not linear with the flow rate or with azimuthal effects. With the restriction to $\gamma=90^{\circ}$, future experimental setups could be designed more suitable and deliver results independent of such an outlet stator.

For technical application, single design aspects could be isolated and examined more in detail in their behavior under inlet or outlet cross-flow. More elaborate modifications of the blade shape, inlet shroud, or guard grille are worth investigating, advisably with a more precisely designed prototype fan instead of industrial fans, with controlled rotation rate and torque. To better understand the flow phenomena caused by cross-flow, optical measurements or numerical simulation of the fan flow field could be undertaken, which may, for example, clarify how design modifications alter the inlet shroud detachment vortex with reference to the influence on the characteristic flow field.

\section{Conflicts of Interest}

The authors declare that they have no conflicts of interest.

\section{Acknowledgments}

The research leading to these results has received funding from the European Union's Seventh Framework Programme (FP7/2007-2013) under Grant Agreement no. 256797, within the "MACCSol" project.

\section{References}

[1] J. S. Maulbetsch and M. N. DiFilippo, "Cost and value of water use at combined cycle power plants," California Energy Commission, 2006.

[2] J. S. Maulbetsch, "Cost/Performance Comparisons of Water Conserving Power Plant Cooling Systems," in Proceedings of the ASME 2011 International Mechanical Engineering Congress and Exposition, pp. 385-390, Denver, Colorado, USA.

[3] G. Kröger, Air-Cooled Heat Exchangers and Cooling Towers, PennWell Books, 2004.

[4] K. Duvenhage and D. G. Kröger, "The influence of wind on the performance of forced draught air-cooled heat exchangers," Journal of Wind Engineering \& Industrial Aerodynamics, vol. 62, no. 2-3, pp. 259-277, 1996.

[5] C. Meyer and A. Zapke, "A modelling strategy for large scale mechanical draught air-cooled systems," in Proceedings of the 10th Int Conf on CFD in oil \&amp; gas, metallurgical and process industries (SINTEF), Trondheim, Norway, 2014.

[6] M. T. F. Owen and D. G. Kröger, "An investigation of air-cooled steam condenser performance under windy conditions using computational fluid dynamics," Journal of Engineering for Gas Turbines and Power, vol. 133, no. 6, Article ID 064502, 2011.

[7] M. T. F. Owen and D. G. Kröger, "The effect of screens on aircooled steam condenser performance under windy conditions," Applied Thermal Engineering, vol. 30, no. 16, pp. 2610-2615, 2010.

[8] C. A. Salta and D. G. Kröger, "Effect of inlet flow distortions on fan performance in forced draught air-cooled heat exchangers," Heat Recovery Systems and CHP, vol. 15, no. 6, pp. 555-561, 1995.

[9] S. J. Van Der Spuy, F. N. Le Roux, T. W. Von Backström, and D. G. Kröger, "The simulation of an axial flow fan performance curve at low flow rates," in Proceedings of the ASME 2011 Turbo Expo: Turbine Technical Conference and Exposition, GT2011, pp. 425-434, Canada, June 2011.

[10] K. Duvenhage, J. A. Vermeulen, C. J. Meyer, and D. G. Kröger, "Flow distortions at the fan inlet of forced-draught air-cooled heat exchangers," Applied Thermal Engineering, vol. 16, no. 8-9, pp. 741-752, 1996.

[11] J. R. Bredell, D. G. Kröger, and G. D. Thiart, "Numerical investigation of fan performance in a forced draft air-cooled steam condenser," Applied Thermal Engineering, vol. 26, no. 8-9, pp. 846-852, 2006.

[12] C. J. Meyer, "Numerical investigation of the effect of inlet flow distortions on forced draught air-cooled heat exchanger performance," Applied Thermal Engineering, vol. 25, no. 11-12, pp. 1634-1649, 2005. 
[13] S. J. van der Spuy, T. W. von Backström, and D. G. Kröger, "An evaluation of simplified methods for modelling the performance of axial flow fan arrays," Research and Development Journal of the South African Institute of Mechanical Engineering, pp. 12-20, 2010.

[14] S. J. van der Spuy and T. W. von Backström, "An evaluation of simplified CFD models applied to perimeter fans in air-cooled steam condensers," Proc Inst of Mech Eng Part A: J Power Energy, no. Part A, pp. 948-967, 2015.

[15] X. Zhang and H. Chen, "Performance Forecast of Air-Cooled Steam Condenser under Windy Conditions," Journal of Energy Engineering, vol. 142, no. 1, Article ID 04015010, 2016.

[16] X. Zhang and T. Wu, "Effects of diffuser orifice plate on the performance of air-cooled steam condenser," Applied Thermal Engineering, vol. 98, pp. 179-188, 2016.

[17] Z. Gu, X. Chen, W. Lubitz, Y. Li, and W. Luo, "Wind tunnel simulation of exhaust recirculation in an air-cooling system at a large power plant," International Journal of Thermal Sciences, vol. 46, no. 3, pp. 308-317, 2007.

[18] C. Butler and R. Grimes, "The effect of wind on the optimal design and performance of a modular air-cooled condenser for a concentrated solar power plant," Energy, vol. 68, pp. 886-895, 2014.

[19] L. Xiao, Z. Ge, X. Du, L. Yang, and Z. Xu, "Operation of aircooling CHP generating unit under the effect of natural wind," Applied Thermal Engineering, vol. 107, pp. 827-836, 2016.

[20] J. S. Maulbetsch, M. N. DiFilippo, and J. O’Hagan, “Effect of wind on air-cooled condenser performance," in Proceedings of the ASME 2011 Int Mech Eng Congr \&amp; Expo, vol. 1, pp. 391396.

[21] J. S. Maulbetsch and M. N. DiFilippo, "Effect of wind on the performance of air-cooled condensers," Final Project Report CEC, California Energy Commission, 2010.

[22] N. Fourie, S. J. van der Spuy, and T. W. von Backström, "Simulating the effect of wind on the performance of axial flow fans in air-cooled steam condenser systems," Journal of Thermal Science and Engineering Applications, vol. 7, no. 2, Article ID 021011, 2015.

[23] G. D. Thiart and T. W. von Backström, "Numerical simulation of the flow field near an axial ow fan operating under distorted inflow conditions," Journal of Wind Engineering \& Industrial Aerodynamics, vol. 45, no. 2, pp. 189-214, 1993.

[24] G. D. Thiart, A Numerical Procedure for Predicting the Effects of Distorted Inflow Conditions on the Performance of Axial Flow Fans [Ph.D. thesis], University of Stellenbosch, 1990.

[25] C. J. Meyer and D. G. Kröger, "Numerical simulation of the flow field in the vicinity of an axial flow fan," International Journal for Numerical Methods in Fluids, vol. 36, no. 8, pp. 947-969, 2001.

[26] W. H. Stinnes and T. W. von Backström, "Effect of cross-flow on the performance of air-cooled heat exchanger fans," Applied Thermal Engineering, vol. 22, no. 12, pp. 1403-1415, 2002.

[27] ISO 5801:2007, "Industrial fans - Performance testing using standardized airways," 2007.

[28] T. Heinemann, C. Bakeberg, H. Lienhart, and S. Becker, “Total pressure measurements behind an axial ventilator using a Kiel probe array," Notes on Numerical Fluid Mechanics and Multidisciplinary Design, vol. 124, pp. 573-581, 2014.

[29] BIPM Joint Committee for Guides in Metrology, "Evaluation of measurement data - guide to the expression of uncertainty in measurement JCGM 100:2008," in GUM 1995 with minor corrections, 2008. 


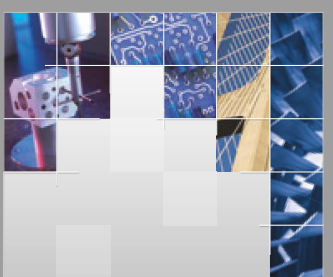

\section{Enfincering}
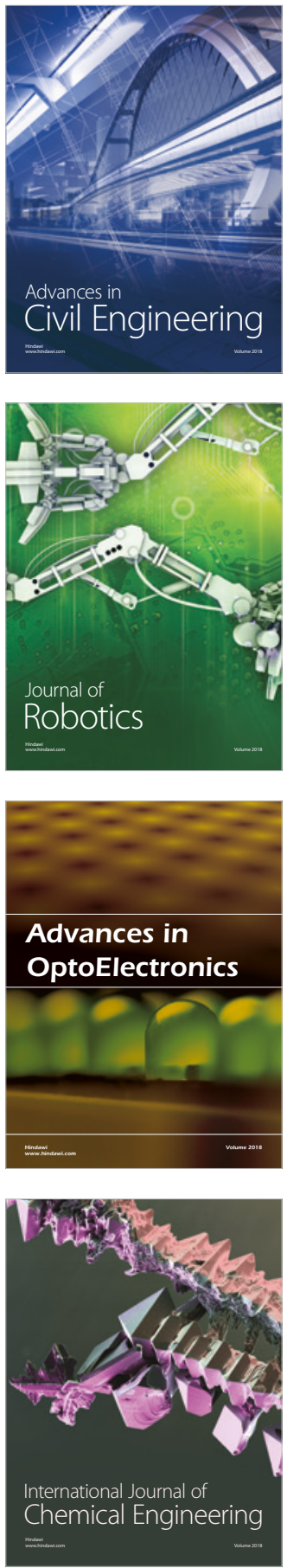

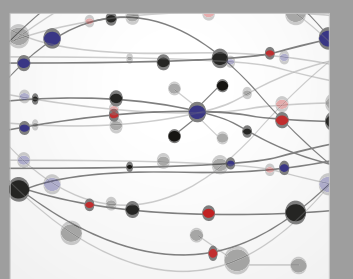

\section{Rotating \\ Machinery}

The Scientific World Journal

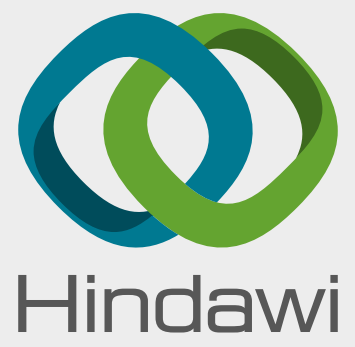

Submit your manuscripts at

www.hindawi.com
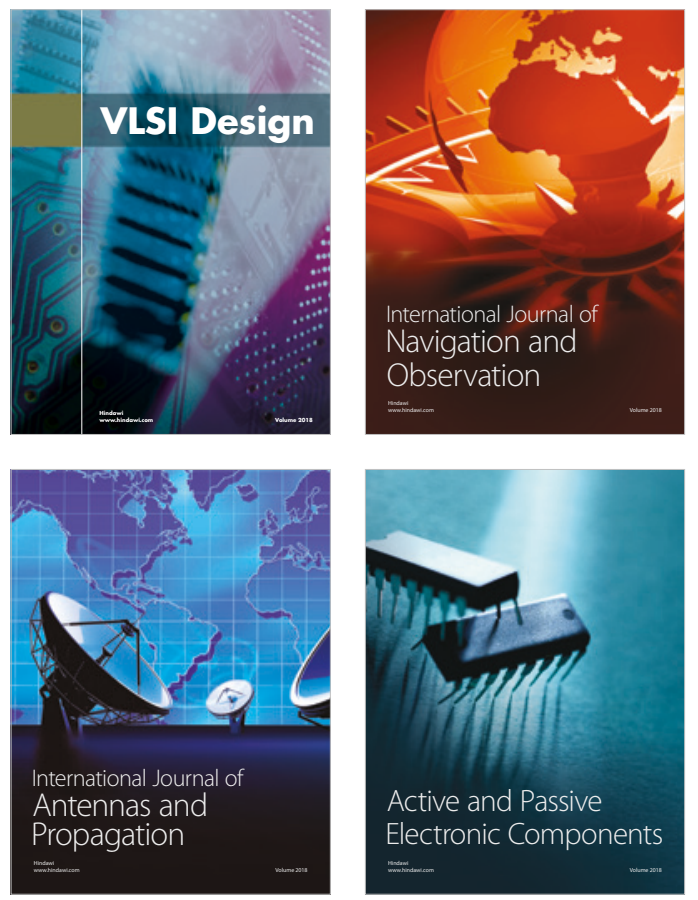
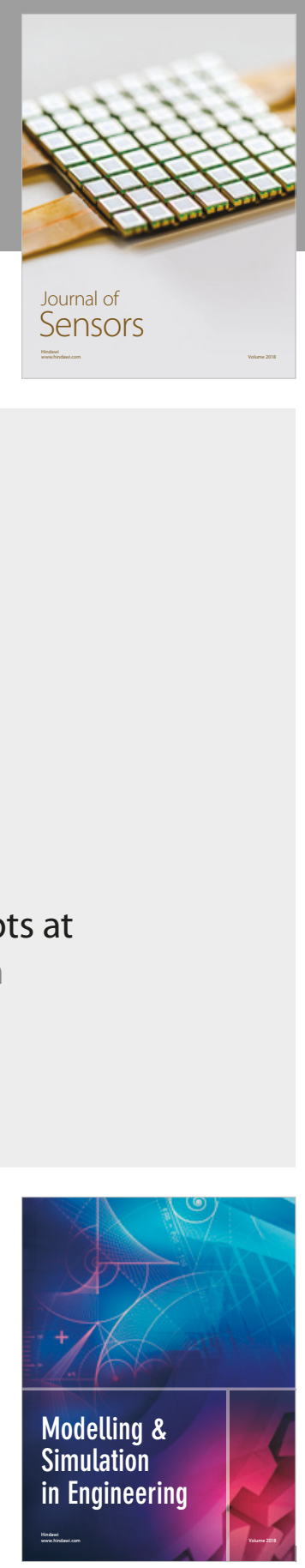

\section{Advances \\ Multimedia}
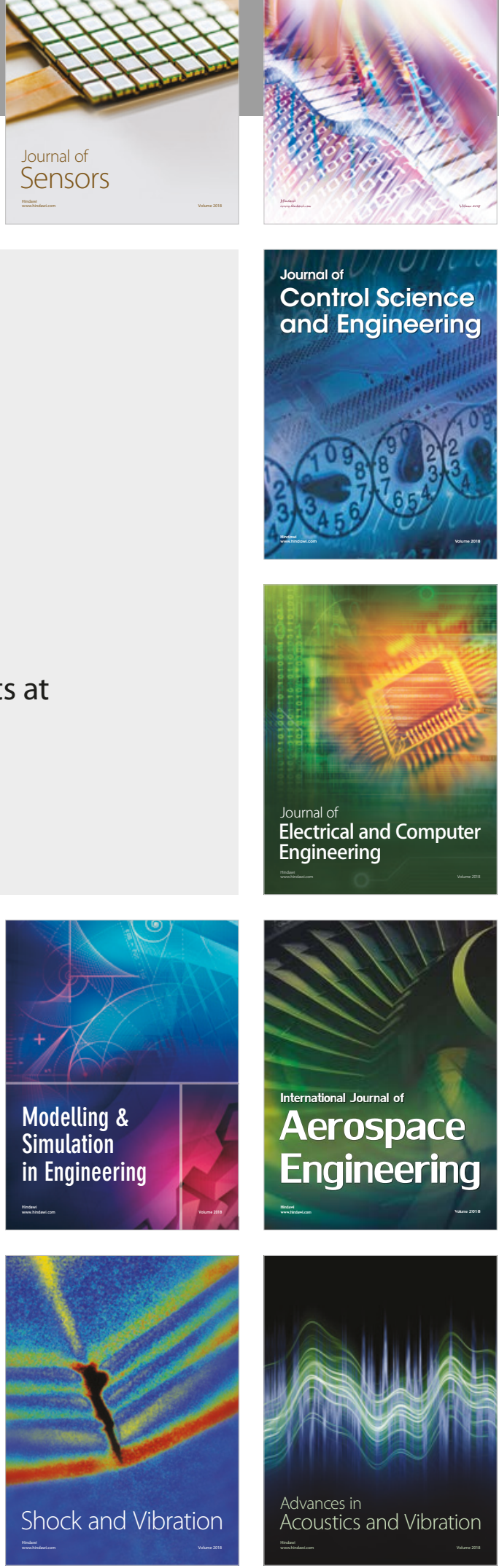